\title{
Translational research into vascular wall function: regulatory effects of systemic and specific factors
}

\author{
Alexander V Maksimenko* \\ Institute of Experimental Cardiology, Russian Cardiology Research-and-Production Complex, Moscow 121552, Russia
}

\begin{abstract}
Development of biomedical techniques has intensified the investigation of endothelial glycocalyx as an individual research object. The effect of vascular wall hydration on progression of pathological lesions, including atherosclerosis, has been revealed and examined. There is evidence that atherosclerosis is associated with water-sulfate and water-sodium exchange. The concept that atherosclerosis is initiated by deficiency of sulfur-containing compounds has been regarded. Glycocalyx protective function against damaging effect of oxidative stress on vascular wall is associated with accumulation and retention of antioxidants on luminal surface of blood vessel. The review deals with protective activity of antioxidant enzyme derivatives, computational methods to determine the principles of the glycocalyx functioning and modeling of the glycocalyx interaction with systemic and specific factors. A limiting influence of crosswind move from clinical practice to life science and contrariwise from life science to clinical practice on the development of translational medicine is emphasized.
\end{abstract}

\begin{abstract}
Abbreviations: BTH: bovine testicular hyaluronidase; CAT: catalase; exSOD: extracellular superoxide dismutase; GAG: glycosaminoglycan; ROS: reactive oxygen species; SOD: superoxide dismutase; SOD-CHSCAT: superoxide dismutase-chondroitin sulfate-catalase conjugate
\end{abstract}

\section{Introduction}

The development of translational medicine increases the effectiveness of therapy and promotes implementation of new pharmacological agents into clinical practice. It should be noted that current achievements of translational medicine do not comply with expectations. This is true for cardiology. Meta-analysis of myocardial infarction studies in a large animal model revealed the following limiting factors hampering successful translation of experimental data to clinical practice: the choice of clinically relevant model and study design [1]. The time of outcome assessment, sex of the animals, and blinding of the operator significantly influence the results obtained and partially account for failures in transition to clinical application. Greater adequacy (anatomically, hemo- and pharmacodynamically, applicability of therapeutic regimens) of large animal models (compared with small animal models) to human body does not abolish the differences. Modeling of accompanying diseases and conditions, such as diabetes mellitus, hypertension, smoking, overweight, etc., in large animals complicates experimental study and considerably increases its cost.

The problem is aggravated by the variety of cardiologic disorders. Thus, therapy of acute conditions, e.g., thromboses, requires elimination of occlusion, an established vascular disorder [2]. This is achieved with plasminogen activators employed in clinical practice as thrombolytic agents [3]. It is noteworthy that plasminogen activator derivatives with a greater molecular weight compared with that of parent substances prevail in the majority of recent laboratory studies. Thrombolytics (Metalyse, Reteplase) currently used in clinical practice have smaller of similar molecular size compared with parent compounds. This discrepancy between clinical data and large body of experimental evidence poses a question about the productive direction of biomedical research, i.e., whether molecular size of plasminogen activator derivatives should be increased or decreased [3]. The validity of the chosen stage/type of acute cardiologic lesion for the success of translational medicine is another aspect for consideration.

Current biopharmacological research is aimed at the development of breakthrough therapies [4] and competition in the most promising areas [5], suggesting that the results of translational medicine are effectively used in clinical practice [6].

Setting of a research goal in the direction from clinical practice to life science (Figure $1 \mathrm{~A}$ ) now predominates in the organization of biomedical studies. This approach is based on various levels of evidence and programs for drug development in clinical medicine and medical research [6]. Simultaneously, transition from life science to medicine can also initiate development of novel drugs (Figure 1 B). Productivity of this approach is strongly determined by the choice of research goal and direction parallel to the development of effective technologies for drug production. Combination of these approaches is successful, judging from the use of thrombolytics based on tissue and urokinase type plasminogen activators as therapeutic agents and development of recombinant DNA technologies for their production [2,3]. Obviously, harmonic balance between the directions from clinical practice to life science (Figure $1 \mathrm{~A}$ ) and from life science to therapy (Figure $1 \mathrm{~B}$ ), their crosswind move enhancing the effectiveness of translational medicine (Figure 1 C).

*Correspondence to: A.V. Maksimenko, 15A $3^{\text {rd }}$ Cherepkovskaya str., Moscow, 121552, Russia, Tel: +7-495-414-60-25, Fax: +7-499-726-31-16, E-mail: alexmak@cardio.ru

Key words: endothelial glycocalyx, vascular wall hydration, water electrolyte balance, antioxidant enzymes, modeled $3 D$ protein structures

Received: February 02, 2017; Accepted: February 20, 2017; Published: February 23, 2017 


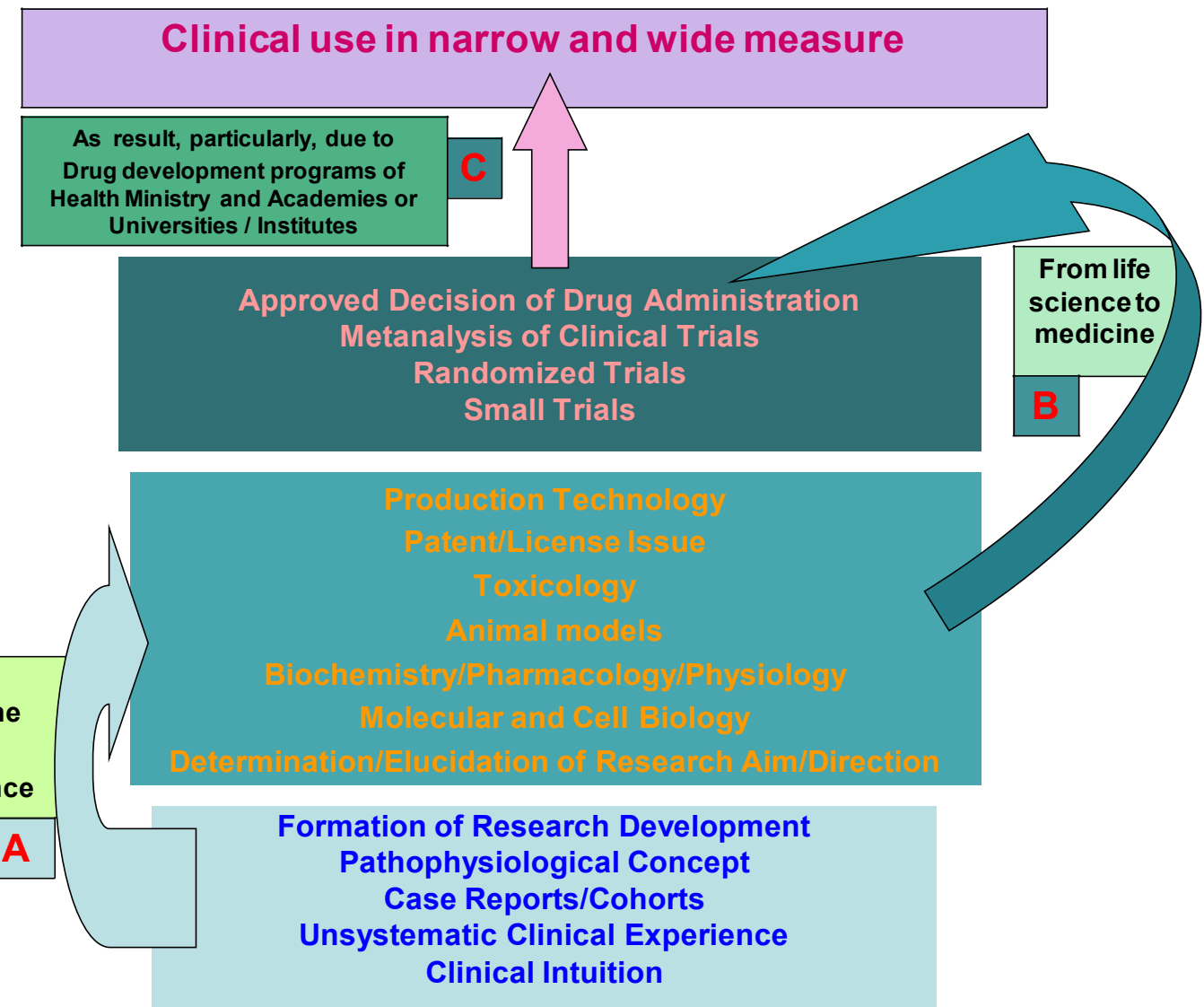

Figure 1. A scheme showing directions of biomedical studies from various levels of evidence and programs for drug development in clinical practice (A) to subsequent preclinical laboratory investigations of this drug (B) followed by its clinical trials and wide-scale production (C).

\section{Primary barrier for preservation of the vascular wall integrity}

Cellular glycocalyx is a functionally significant component in the circulatory system $[7,8]$. Interest in the investigation of glycocalyx has increased with improvements of laser scanning microscopy techniques which allow reconstruction of visualized objects and their accurate analysis. Glycocalyx with a thickness of $2-3 \mu \mathrm{m}$ was visualized on the surface of cultured endotheliocytes by the method of confocal scanning microscopy [9]. In murine carotid arteries glycocalyx (thickness 3.5$5.5 \mu \mathrm{m}$ ) was visualized by two-photon microscopy [10]. Glycocalyx is represented by an outer layer of macromolecules, predominantly protein-carbohydrate and carbohydrate-lipid complexes bound to the plasma membrane and together with blood plasma proteins forming a lining with specific structure and a vast range of functions $[7,8,11]$ (Figure 2). Noninvasive diagnostic methods have been tested to analyze the relationship between the state of endothelial glycocalyx and cardiovascular risk in humans. The analysis employs data obtained with the use of orthogonal polarization spectroscopy (OPS) [12] and sidestream dark field imaging (SDF) [13]. A reverse relationship between the thickness of endothelial glycocalyx in the microcirculatory network and the risk of cardiovascular lesions has been established. The collected body of evidence indicates that endothelial glycocalyx is a potential barrier between health and vascular disease [14], suggesting that it can be used as diagnostic marker and therapeutic target in cardiovascular medicine [15]. Thus, luminal surface of the vascular wall has a double protective endothelium/glycocalyx lining, glycocalyx being the first defense barrier against vascular injury.

\section{Destruction of glycocalyx and pathological factors}

Regarded as an individual vascular endothelial surface layer, normal glycocalyx is a self-renewing 3D network in dynamic equilibrium with circulating blood. Glycocalyx involves a vast array of functions, such as a vascular permeability barrier and molecular sieve, mechanical transductor of blood flow shear stress, leukocyte and platelet adhesion regulation, binding of chemokines and growth factors, deposition of vasoactive agents and coagulation factor inhibitors, and realization of their functions is of great importance for the maintenance of blood plasma and vascular wall homeostasis $[15,16]$.

Glycocalyx destruction is an initial event in the vascular lesion development caused by pathological factors. It is an essential initial stage in diabetes mellitus-related micrangiopathies and chronic venous diseases [17]. Ischemia/reperfusion, infection, diabetes and renal failure induce glycocalyx destruction [18-20]. A decrease in its volume occurring in hyperglycemia increases vulnerability of blood vessels and is associated with endothelial dysfunction (elevation of plasma hyaluronan) and coagulation activation in vivo (high blood contents of 1+2 prothrombin fragment and D-dimer of fibrin) [21]. Various pathological factors (hypercholesterolemia, inflammation, hyperglycemia, excess of salts in the body, shear stress, nephrotic syndrome, etc) destroy glycocalyx [22-24]. It was suggested that glycocalyx destruction detected as changes in its composition and a decrease in its thickness has an important role in the development of endothelial dysfunction [22,25] and develops in patients with acute coronary syndrome [26]. The diversity of pathological factors inducing glycocalyx destruction that leads to progression of various 


\section{BLOODSTREAM}

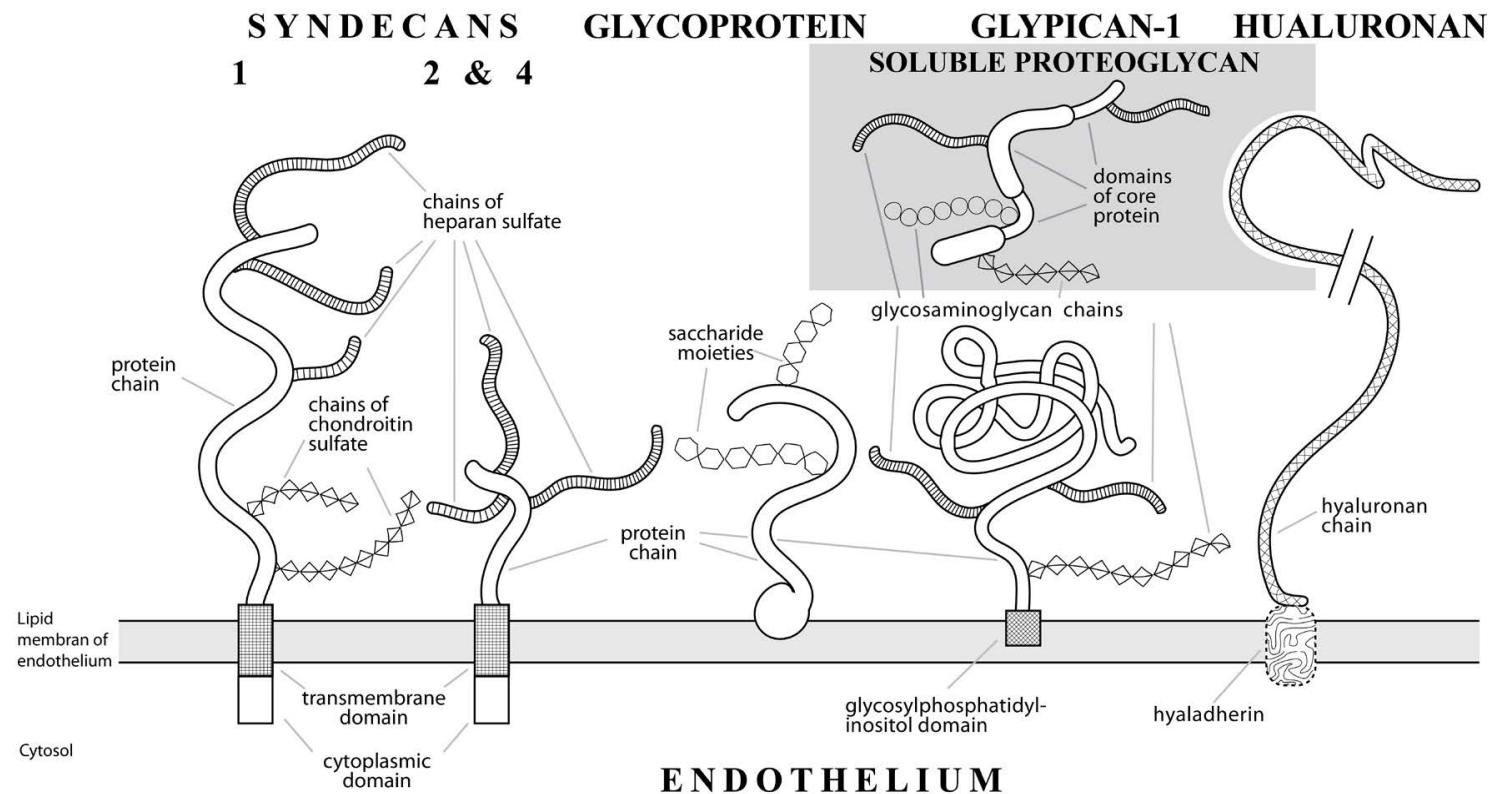

Figure 2. A schematic drawing of membrane-bound (syndecans and glypican-1) and soluble (given in general form) proteoglycans of endothelial glycocalyx with hyaluronan and glycoproteins on the luminal surface of a blood vessel. Proteoglycans from syndecan group (with a transmembrane protein domain) are divided into 4 subtypes according to the composition of glycosaminoglycan chains. Glypican group (bound to plasma membrane via glycosylphosphatidylinositol domain) includes 6 subtypes according to the bond type and the number of glycosaminoglycan chains. N-terminal segment of glypican-1 (directed to the lumen) contains numerous cysteine residues which determine globular shape in contrast to elongated extracellular domains of syndecans. Endothelial glycocalyx proteoglycans are classified according to various parameters (species origin, composition and number of glycosaminoglycan chains, core protein type, etc.), combined according to their function in the body: coupling of extra- and intracellular signals of endothelial function.

diseases implies a common stage in the genesis of circulatory disorders followed by various pathologies.

\section{Hydratation and dehydratation of the vascular wall}

In normal tissues, extracellular (matrix, interstitium) and juxtacellular (glycocalyx) gel components are maintained under relatively dehydrated conditions [27]. This facilitates proper circulation and vascular permeability. Hydration volume of gel matrix is regulated by equilibrium of various forces, including elasticity of polymer components, their chemical affinity, fixed charge, and osmotic interactions, being dependent on glycosaminoglycans, i.e., mechanical and structural factors responsible for their content and spatial distribution in intact tissues.

Macromolecular interactions which control and determine atherogenesis are aimed against dehydration forces that prevail in vivo in the norm. Indeed, shift of the dehydration/hydration equilibrium towards hydration occurs upon glycocalyx destruction caused by various pathological factors (Figure 3) [28,29]. This, in turn, affects hydration volume, modifying glycosaminoglycan structure and distribution [27]. Glycocalyx integrity plays an important role in tissue protection against edema. Under pathological conditions (inflammation, thrombosis, infection, etc.) hydration manifests itself as edema of arterial tissues, which hampers the flow of nutrients and drugs [28]. Diffusional thickening and fibril disorganization in the intima occurring in atherosclerosis are associated with changes in local water homeostasis [27].

These findings show that dehydration and hydration of endothelial glycocalyx are important parameter of normal and deceased vascular wall. This is supported by the fact that the body of an adult with good cardiopulmonary health consists of $60 \%$ water [30]. Two thirds of this volume is localized intracellularly, one third, extracellularly.
Interstitium contains $80 \%$ of this volume; other $20 \%$ is part of blood plasma volume.

\section{Water-sulfate exchange}

Sulfation of glycosaminoglycans is a specific factor affecting water balance in the glycocalyx. Alterations in this parameter are associated with the volume of solvent available/required for hydration [27,31]. Hydration of chondroitin sulfate increases with progression of atherosclerotic lesions, which decreases its anticoagulant activity (antithrombin activation) [26] and increases glycocalyx hydration. Insertion of ${ }^{35} \mathrm{SO}_{4}$ in glycosaminoglycans of chicken glycocalyx was slowly $(12 \mathrm{~h})$ suppressed by the tetrasaccharide hyaluronan and its derivatives of higher molecular mass [32]. Total evidence indicates that in blood vessels with atherosclerotic plaques sulfate insertion is linked to accumulation of sulfated glycosaminoglycans in the atheroma for their redistribution. This can be regarded as an indirect preparation for an increase in vascular area with low sulfation suitable for a potential lesion [33]. Sulfated glycosaminoglycans maintain zones of structured water which are necessary for normal circulation (condition of the endothelium and motion of red blood cells along capillaries). Sulfur deficiency in the body can contribute to a decrease in cholesterol sulfate level and glycosaminoglycan sulfation [33]. Loss of sulfates provokes changes in structural water that lead to accumulation of cholesterol in atheroma, since its transport in water-based media depends on sulfation, i.e., cholesterol transport disorders are related to low bioavailability of sulfate in the circulation. It has been hypothesized that low cholesterol sulfate content together with decreased sulfation of glycosaminoglycans in the body is a key lesion propulsive to atherosclerosis progression [33].

\section{Water-sodium exchange}

Negative charge on the vascular wall (created predominantly by 


\section{PATHOLOGY FACTORS DESTRUCTIVE}

\section{ENDOTHELIAL GLYCOCALYX}

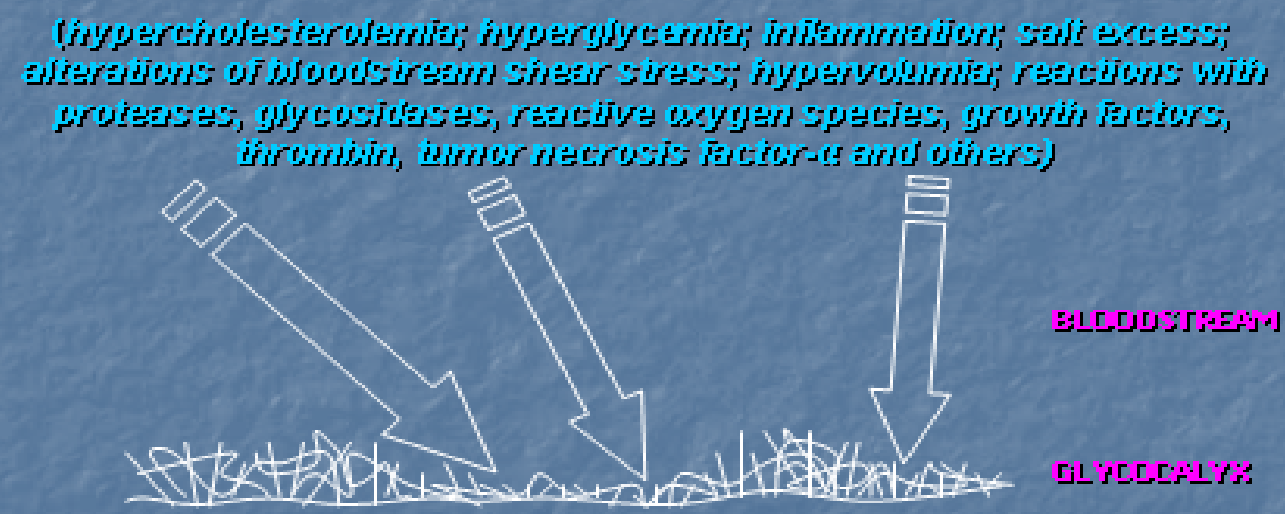

EIIUSjSEI

드는

Figure 3. A scheme illustrating destruction of endothelial glycocalyx leading to the development of atherosclerosis, diabetes mellitus, sepsis, damage after ischemia/reperfusion, tumor angiogenesis, metastase growing and acute pulmonary disease.

the glycocalyx) and on circulating blood components e.g., red blood cells facilitates frictionless blood flow. Removal of the surface negative charge (as a result of enzyme reaction) enhances red blood cell adhesion to endothelial cells [34]. A similar effect is produced by sodium cation. At high physiological concentrations $(>140 \mathrm{MM}) \mathrm{Na}^{+}$saturates surface negative change, thus increasing adhesion. At $\mathrm{Na}^{+}$concentrations $<140 \mathrm{MM}$ the number of vacant negative charges remains sufficient to maintain adhesion of red blood cells to endothelial lining at a low level. Sodium renders endothelium sticky for red blood cells [34]. Storage of plasma $\mathrm{Na}^{+}$in the glycocalyx (with partial neutralization of negative surface charge) allows one to divide glycocalyx into "good" which has a capacity for $\mathrm{Na}^{+}$accumulation to maintain sufficient negative charge at normal plasma $\mathrm{Na}^{+}$concentration and "bad" when the loss of negative charge increases adverse interactions between endothelium and red blood cells [35]. Deeper penetration of red blood cells to endothelial glycocalyx is associated with impaired microvascular perfusion [36]. The concept of "good" and "bad" glycocalyx laid the basement for salt blood test when quantitative determination of red blood cell sodium allows indirect assessment of the luminal vascular wall state [35]. Irrespective of treatment, hypertensive patients had a high salt sensitivity according this test. Interestingly, at a mean $\mathrm{Na}^{+} /$ $\mathrm{K}^{+}$selectivity in the glycocalyx of red blood cells equal to $6.1 \pm 0.39$ their adhesion to endothelial surface is 180:1 [37]. Thus, low concentration of sodium cations maintains frictionless passage of red blood cells through capillaries, while preservation of endothelial glycocalyx is important for maintaining balanced function of the vascular system [35,37].

The data on water-sulfate and water-sodium exchange stress the significance of water-electrolyte balance for maintaining the function of endothelial glycocalyx and vascular wall. Further research in this direction is prospective for elucidation of new regulatory mechanisms in the cardiovascular system.

\section{Glycocalyx and reactive oxygen species}

The multiform interaction between systemic factors such as endothelial glycocalyx and oxidative stress in blood circulation emphasizes and grounds the signification and perspective for consecutive development of novel prevention and therapy approaches for sustention and cure of cardiovascular human health.

Glycocalyx is involved in oxygen metabolism in the circulatory system. At moderate concentrations reactive oxygen species (ROS) participate in cell signal transduction, while at higher concentrations they produce damaging effects [38]. Endothelial dysfunction as a key initial stage in the development of atherosclerosis can be associated with high ROS production or oxidative stress which is a major pathogenic factor of this process. A common mechanism of damaging effect renders oxidative stress a dominating factor in the development of various cardiovascular disorders [39,40]. Excessive ROS generation leads to destruction of endothelial glycocalyx [41]. To prevent and reduce this damage the glycocalyx accumulates antioxidants e.g., extracellular superoxide dismutase (exSOD, Figure 4). This enzyme has high affinity for heparansulfates of endothelial glycocalyx due to a specific positively charged binding domain for heparin-like compounds and high expression (up to $70 \%$ total vascular superoxide dismutase / $\mathrm{SOD} /$ content) [11]. It was reported that glycocalyx has a role in $\mathrm{NO}$ and ROS production [42]. Ex vivo incubation of femoral porcine arteries with hyaluronidase to remove hyaluronan decreased both nitrite content and vasodilation. Degradation of heparansulfateproteoglycan and syalic acid by heparinase III and neuraminidase, respectively, reduced NO bioavailability by increasing superoxide production. This finding indicates that hyaluronan is involved in shear stressinduced NO production and heparansulfate together with syalic acid participates in ROS production in response to shear stress. Since a vast 

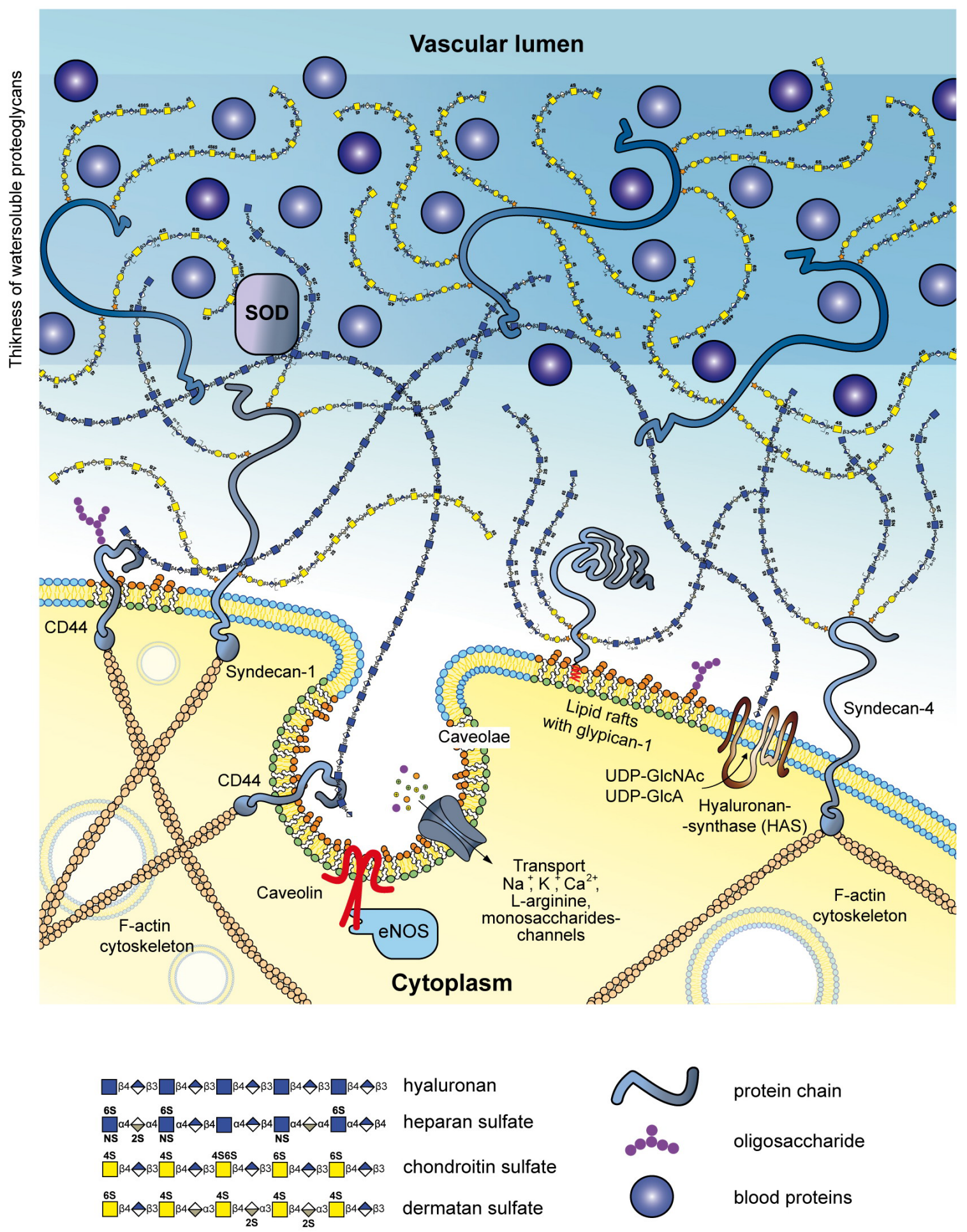

Figure 4. A hypothetic scheme showing the major glycocalyx components on the endothelial surface.Location of extracellular superoxide dismutase (SOD) which displays affinity for endothelial heparansulfate structures is indicated. Heparansulfate fulfills protective function by accumulating antioxidants, including extracellular SOD which converts cytotoxic superoxide radical into hydrogen peroxide. 
majority of cardiovascular disorders is associated with oxidative stress, its damaging effects should be blocked or reduced. Endogenous and exogenous antioxidants can be employed for this purpose.

\section{Formation of antioxidant therapy}

Clinical studies of antioxidants test the hypotheses that low contents of glutathione peroxidase and superoxide dismutase increase the risk of major adverse cardiovascular events [43], modified expression and/or activity of antioxidant enzymes is associated with oxidative stress which is an important factor in the pathogenesis of age-related (macular degeneration) and degenerative diseases [44], as well as evaluate the effectiveness of antioxidant therapy and identify unfavorable effects of oxidative stress on specific clinical parameters of cardiovascular therapy $[39,40]$.

Formation of antioxidant therapy implies investigation and development of new antioxidant drugs $[45,46]$. Protective effects of ascorbic acid, trolox, melatonin, polyphenols [47], human recombinant Mn-SOD [48] in hepatic disorders, the effects of chemically modified SOD in lipid oxidation and antioxidant status in diabetic rats [49], changes in SOD and catalase (CAT) activities due to cadmium-induced incorrect folding [50] have been examined. Antioxidant enzymes remain an important object of biomedical research.

Superoxide dismutase and catalase (up to 30\% total content) were precipitated on a protective antioxidant nanocarrier for endothelial targeting and bound to antibodies against endothelial adhesion molecules to provide the targeting [51]. In a mouse model of endotoxin-induced pulmonary damage CAT derivative protected endothelium, decreased lung edema and leukocyte infiltration. SOD derivative reduced cytokine-induced proinflammatory activation of the endothelium and endotoxin-induced pneumonia. A modular approach has been suggested for targeting therapeutic enzymes at the vascular wall [51]. Among three concentration intervals of reactive forms of oxygen and nitrogen species (physiological, enhanced and toxic), there is the favourable effect on toxic interval with such approaches as center-specific, nanocarrier-based therapy [52], 3D supramolecular ensembles with antioxidants or enzymes [53] and nanotherapeutics with an antioxidant nucleus for blocking atherogenic oxidative stress and an amphiphilic polysaccharide covering targeted at scavenger receptor to inhibit athero-inflammatory cycle [54]. It should be noted that the effectiveness of blocking all ROS requires cooperative action of antioxidants [55], including synergist combinations of antioxidant enzymes, as exemplified by the original bienzyme conjugate superoxide dismutase-chondroitin sulfate-catalase (SOD-CHS-CAT) [56].

\section{Bienzyme conjugate of antioxidant biocatalysts}

Covalent binding of superoxide dismutase and catalase via chondroitin sulfate in the SOD-CHS-CAT conjugate provides a simultaneous action of these enzymes in the damaged area. The antithrombotic effect in vivo produced by the conjugate is higher than those of various combinations and mixtures of its components (native or modified) [46,56], is achieved at low doses (25-50 U SOD and 55-110 $\mathrm{U}$ CAT per rat), which is one or two orders of magnitude lower than those for native or modified enzymes [57]. Chondroitin sulfate content on the vascular surface over atherosclerotic lesions increases at the initial stages of atherogenesis [58]. Intimal thickening is associated with the presence of versican, a proteoglycan with chondroitin sulfate and dermanan sulfate chains [59]. Versican is accumulated in the neointima of human blood vessels prone to atherosclerosis [60]. Specific features of chondroitin sulfate [58-60] render it into a productive linking agent for antioxidant enzymes [45] and facilitate targeting of the resulting conjugates at the glycocalyx over vascular lesions. This may account for the effectiveness of SOD-CHS-CAT conjugate at small doses [45,46]. It should be noted that in an animal model of endotoxic shock SOD-CHSCAT significantly increases viability after preventive and therapeutic administration [56]. These results are interesting in comparison with modeled therapeutic effects of modular approaches [51] and for a better understanding of the mechanism underlying the conjugate activity involving NO-dependent and NO-independent pathways for its therapeutic effect. The effectiveness of SOD-CHS-CAT conjugate urges further development of genetic and bioengineering technologies for production of three-functional enzymes with activities of SOD and CAT [61] and peroxidase and SOD [62] capable of entering the cell.

Damaging effect of oxidative stress on the vascular wall is determined by a complex of multivariate factors. An array of variables should be taken into account to evaluate and control this effect, which actualizes the use of computational methods to optimize the solution of the specific biomedical problems.

\section{Computational studies of systemic factors and their regulators}

Calculated interactions (computational models) have been used for several decades to evaluate and predict function of biological systems, e.g., fibrinolysis, coagulation, etc. This approach, however, implies a complex processing and presentation of the data obtained with a wide experimental verification gap. Nevertheless, the significance of computational methods increases as new systemic factors are considered for achieving the goals set up by translational medicine. Current methods are oriented toward experimental verification and cover general objects of analyzed systems. Variations in the microcirculation were studied using modeled glycocalyx-endotheliumerythrocyte interactions [63]. These variations are determined by vascular wall modifications associated with endothelial cell shape, glycocalyx-related effects and specific parameters of the blood. Blood is modeled as a two-component (plasma and corpuscular elements) incompressible fluid. Endothelial glycocalyx is modelled as a medium of variable and adaptive porosity manifesting itself in the magnitude and temporal variations of blood flow rate and its shear stress. Combined and simultaneous effect of endothelial wall undulation, glycocalyx compression and repulsion, and specific nature of the blood on the flow properties has been demonstrated [63].

Structural reconstructions of components and external agents that can influence biological system have been performed to analyze the state of the vascular wall. Investigation of the mechanism responsible for specific interactions between glycan-binding protein with cognate glycan from cell glycane array (glycome) was initiated with the use of automated 3D structure generation technique (computational carbohydrate grafting) [64]. Integration of extensive data from glycan screening and protein crystallography facilitates construction of putative co-complex structures that can be objectively assessed and iteratively altered until a high level of agreement with experiment is achieved. This approach allows differentiation of active glycan determinants from potential ones. When applied to a collection of 10 protein-glycan complexes, for which crystallographic and array data have been reported, grafting provided structural rationalization for the binding specificity of $>90 \%$ of 1223 arrayed glycans [64]. Automated molecular docking and interaction mapping techniques were employed to characterize glycosaminoglycan-protein interactions [65]. The method was used for identification of glycosaminoglycans 
(GAG) capable of binding to acidic fibroblast growth factor. The results obtained demonstrate the value of mapping-based techniques in identifying specific GAG epitopes recognized by proteins and for GAGbased drug design. Combinatorial virtual library screening technology was used to predict high-specificity GAG sequences, bearing in mind their phenomenal structural diversity [66]. The results highlight critical interactions in heparin/heparansulfate oligosaccharides that regulate specificity, the minimal specificity element being a disaccharide of heparin.

The antioxidant enzymes catalase, superoxide dismutase, glutathione peroxidase and glutathione reductase act as defense agents against oxidative stress. Three-dimensional structures of these enzymes were modeled and docked with nonylphenol and bisphenol A which induce oxidative stress [67]. The study revealed that the enzymes have binding sites for nonyphenol and bisphenol. Maximum inhibition by nonyphenol was demonstrated for catalase and by bisphenol A for superoxide dismutase. These findings specify the conditions for the use of SOD and CAT. Microcirculation disturbances can be controlled by modifying the state of the glycocalyx with enzymes, for example, hyaluronidase [68]. Effects of hyaluronidase in native and chondroitin sulfate modified forms demonstrated the regulation potential for microcirculation recovery after ischemia/reperfusion tracked using laser Doppler flowmetry. This action is actual for cardiovascular therapy of acute myocardial infarction, thromboses, no-reflow [69]. However, scarce information about the structure of these biocatalysts hampers the research into regulation of the glycocalyx state. To solve this issue a 3D-model of bovine testicular hyaluronidase (BTH) [70] based on established tertiary structure of human hyaluronidase [71] was constructed using a molecular homological modeling method in silico (Figure 5). Superposition of BTH with human and bee venom hyaluronidases has revealed differences between these enzymes [70].
Human hyaluronidase has a Ser-353 - Trp-435 C-terminal domain homologous to epidermal growth factor [71] which is present in our model of BTH (Figure 5) and bee venom hyaluronidases. Epidermal growth factor domain has three disulphide bonds, is essentially stable, regulates binding with other proteins and modulates the activity of a full-size enzyme [70,71]. A 3-D structural model of chondroitin-modified BTH was constructed by modeling covalent binding between lysine residues of the enzyme and benzoquinoneactivated chondroitin sulfate links (Figure 6). The significance of deep modifications of BTH for production of its active and stable derivatives was demonstrated by varying the degree of the enzyme modification and the size of covalently bound chondroitin sulfate chains and was confirmed experimentally [70]. Effective size of chondroitin sulfate coating is achieved with $180-\mathrm{kDa}$ enzyme derivatives obtained with the use of $30-50-\mathrm{kDa}$ and/or $120-140-\mathrm{kDa}$ chondroitin sulfate. Lysine residues on the surface of $\mathrm{BTH}$ have different bioavailability: there are external free and semifree residues (their modification affects catalytic domain) and cryptic residues [70]. It should be noted that epiphycan effectively binds to lysine residues of type I, III, VII, VIII and $\mathrm{X}$ collagen via clustered chondroitin sulfate, while the affinity of binding to type II, IV, V, VI and IX collagen displays low affinity [72]. Chemical modification of collagen lysine residues decreases binding affinity for clustered chondroitin sulfate and determines binding affinity for epiphycan. Examination of a 3-D modeled initial stage of docking of $\mathrm{BTH}$ with chondroitin sulfate trimer or heparin tetramer revealed several surface binding sites for GAG on the enzyme molecule. Both reversible and irreversible conformational changes depending on location of other negatively charged GAG ligands on the protein globule were demonstrated. In irreversible conformational changes, Glu-149 (E 149) and Asp-147 (D 147) residues that play a key role in enzyme activity move from the active center to the periphery, which causes inactivation of the enzyme (Figure 7). These findings suggest

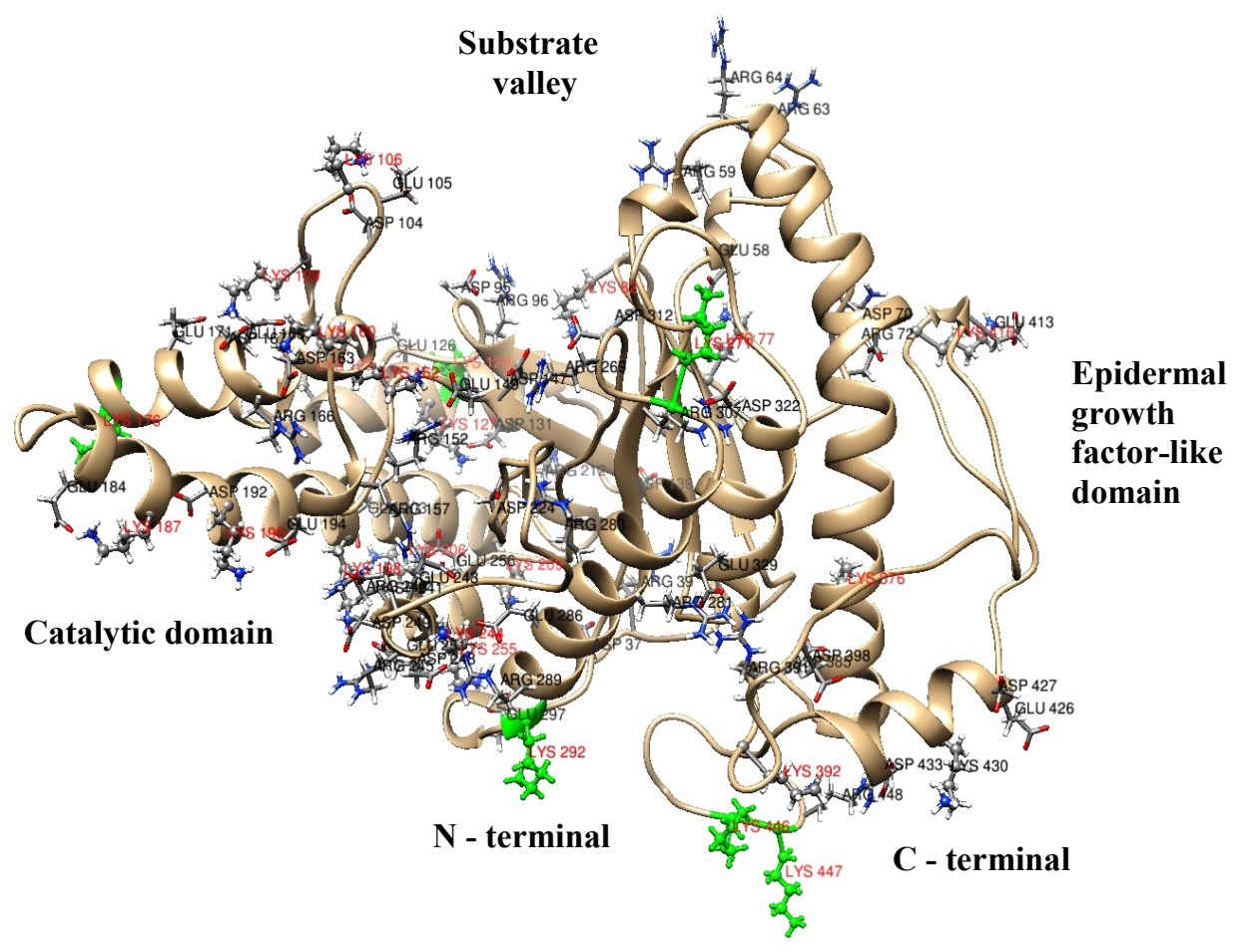

Figure 5. Illustrated visualization of a 3-D model of bovine testicular hyaluronidase. The localizations of lysine and asparagine residues and glutamic and asparaginic amino acids are indicated. Six lysine residues of the first level availability (external free amino acids) are highlighted green. 


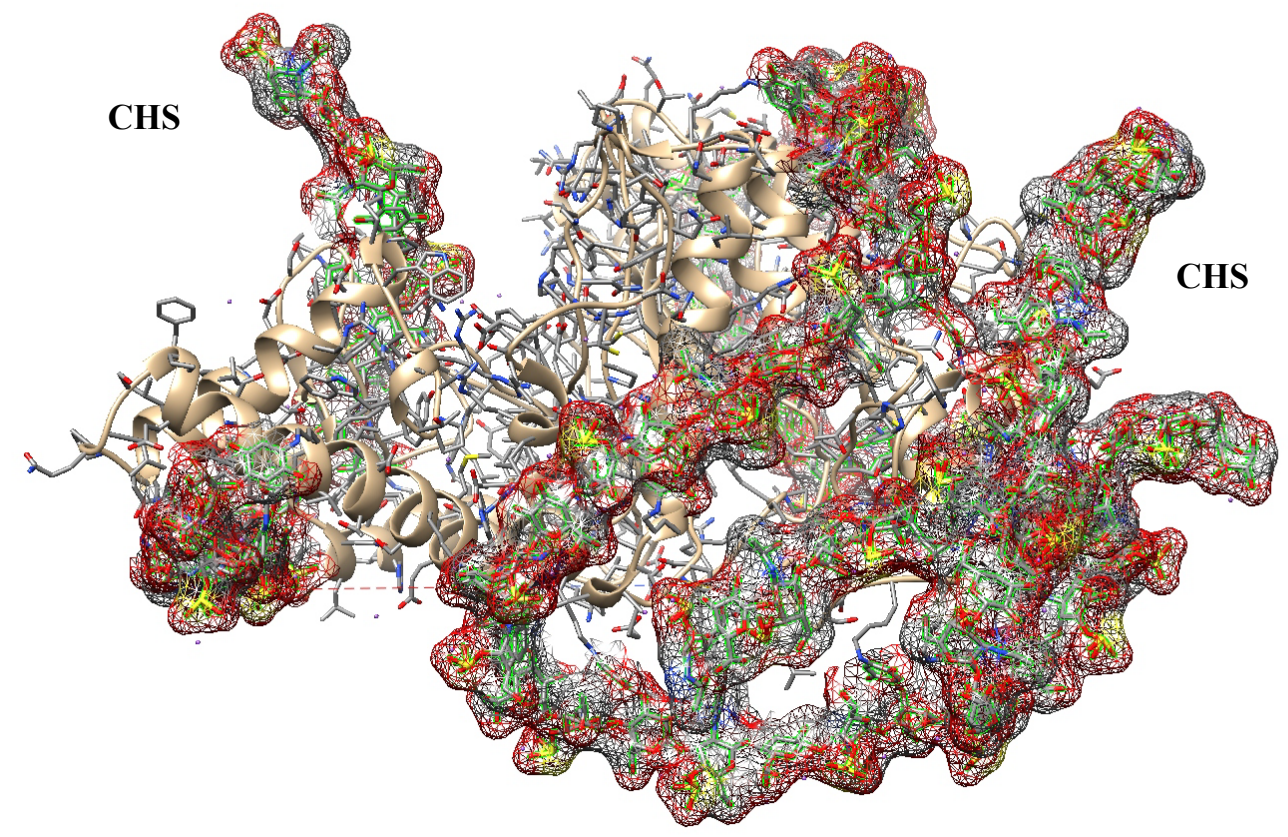

Figure 6. Illustrated visualization of 3-D model of bovine testicular hyaluronidase covalently modified by two chondroitin sulfate chains (designated as CHS and highlighted gray, red and brown). The chains are necessary for deep modification of the enzyme with binding at its 19 lysine residues (positions 77, 106, 122, 129, 176, 187, 190, 198, 206, 244, 255, 271, 292, 376, $392,416,430,446,447)$. Enzyme structure shown as a ribbon sort and as segment forms is masked by covalently bound chondroitin sulfate chains (30 kDa).

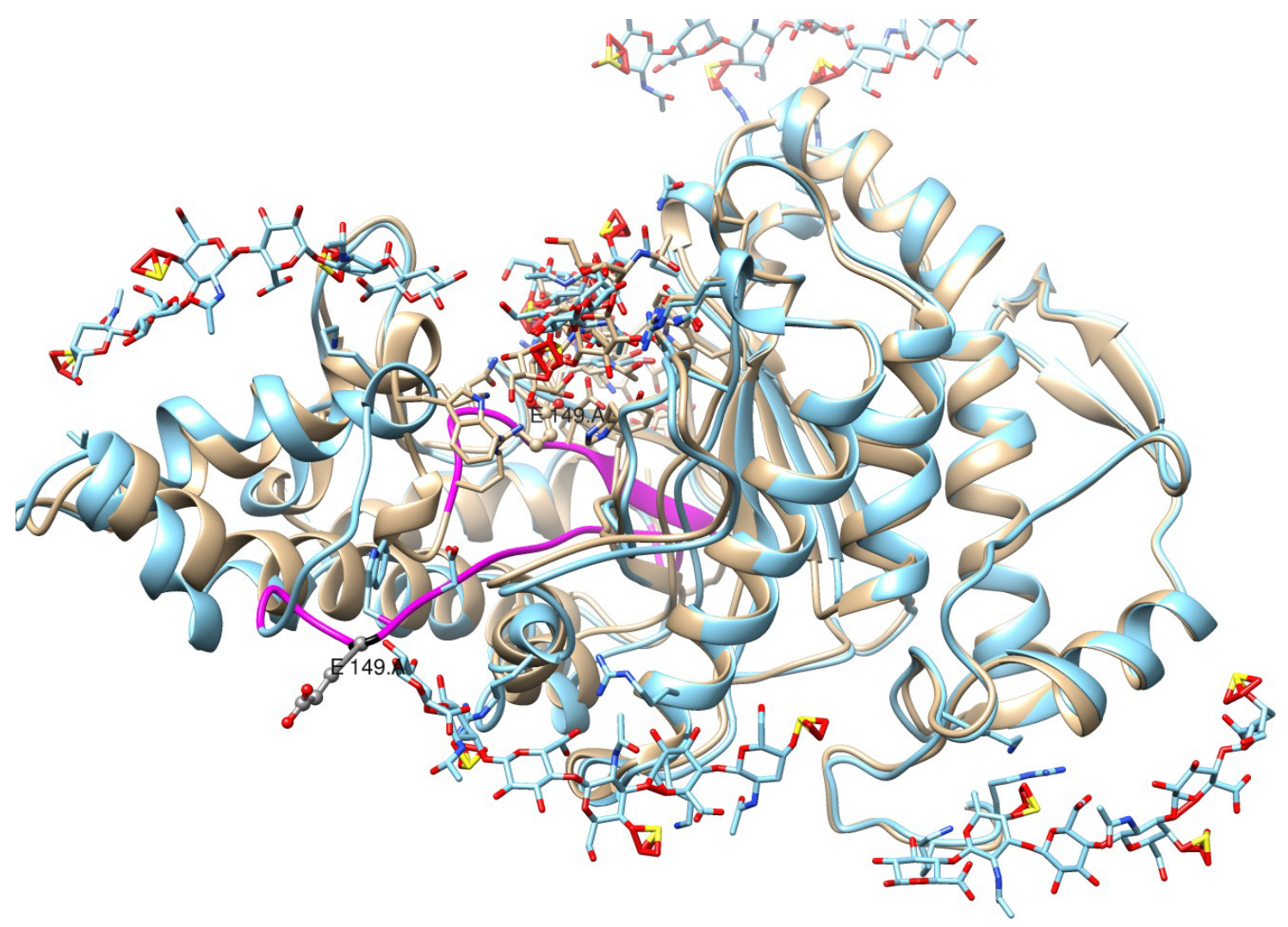

Figure 7. An irreversible conformational change in bovine testicular hyaluronidase with Glu-149/E 149 and Asp-147/D 147 moved to the periphery of enzyme structure and considerable alterations in protein loop/active center chain (highlighted violet). Location of enzyme structures on the 40 th $p s e c$ (beige) and on the $160^{\text {th }}$ psec (blue) of molecular dynamics induced by glycosaminoglycan ligands. 
some threshold interactions and set the goal of their identification to obtain control over hyaluronidase function on the vascular wall.

Presented above data have been showed the alterations of our knowledge in respect to functioning of multicomponent biological systems with application of computational methods and with consecutive ascertainment of approaches for regulation of their state.

\section{Conclusion and future perspectives}

Extensive systematic studies of the glycocalyx have revealed specific factors limiting its function. Better understanding of the mechanisms responsible for regulation of glycocalyx hydration opens new perspectives in the study of initial vascular wall damage related to water-electrolyte balance disorders. Further progress in this study is determined by clinical data. Control over the interaction of factors in extensive systems such as vascular glycocalyx and oxidative stress offers productive approaches in antioxidant therapy and highlights the role of antioxidant enzymes in correction of metabolic disorders. Computational methods for multicomponent systems proved to be useful for therapeutic regulation of the vascular system function and provide a better understanding of the principles of endothelial glycocalyx function with the development of approaches to their effective use. Experimental findings largely determine progress in this direction in largely. Fundamental insight into molecular and cellular mechanisms operating in the vascular system could contribute to translational cardiology. Clinical and biochemical research into vascular wall hydration and water electrolyte balance provides new insights in the mechanisms underlying the initiation of vascular lesions, their prevention and therapy. Consecutive study of the glycocalyx function, computational research of its relationship with oxidative stress, and investigation of the glycocalyx state regulation is aimed at the development of novel approaches to the vascular wall protection. Successful development of translational medicine depends on close cooperation between scientists and clinicians as well as on constantly increasing data flow from life science laboratories to clinics and vice versa. This crosswind move is a determinant factor of efficacy for translational cardiology.

\section{Acknowledgements}

The study was financially supported by Russian Foundation for Basic Research (grant 15-04-03584) and Health Care Ministry of Russian Federation.

\section{References}

1. VanHout GPJ, Jansen of Lorkeers SJJ, Wever KE, Sena ES, Kouwenberg LHJA, et al. (2016) Translational failure of anti-inflammatory compounds for myocardial infarction: a meta-analysis of large animal models. Cardiovasc Res 109: 240-248. [Crossref]

2. Van de Werf F (2014) The history of coronary reperfusion. Eur Heart J 35: 2510-2515. [Crossref]

3. Maksimenko AV (2012) Cardiological biopharmaceuticals in the conception of drug targeting delivery: practical results and research perspectives. ActaNaturae4: 72-81. [Crossref]

4. Scott C (2015) Speeding development and lowering cost while enhancing quality: a BPI theater roundtable at the 2015 BIO Convention. Bioprocess Int 1 (Suppl 4): 23-25.

5. Hoefer IE, Steffens S, Ala-Korpela M, Bäck M, Badimon L, et al. (2015) On behalf of the ESC Working Group Atherosclerosis and Vascular Biology. Novel methodologies for biomarker discovery in atherosclerosis. Eur Heart J 36: 2635-2642. [Crossref]

6. Lüscher TF (2013) The bumpy road to evidence: why many research findings are lost in translation. Eur Heart J 34: 3329-3335. [Crossref]

7. Reitsma S, Slaaf DW, Vink H, van Zandvoort MAMJ, ondeEgbrink MGA (2007) The endothelial glycocalyx: composition, functions, and visualization. Pflügers Arch 4: 345-359.[Crossref]
8. Maksimenko AV, Turashev AD (2014) Endothelial glycocalyx of blood circulation system. I. Detection, components, and structure organization. Russ J BioorgKhim 40 : $119-128$

9. Barker AL, Konopatskaya O, Neal CR, Macpherson JW, Whatmore JL, et al. (2004) Observation and characterization of the glycocalyx of viable human endothelial cells using confocal laser scanning microscopy. PhysChemChemPhys 6: 1006-1011.

10. Megens RT, Reitsma S, Schiffers PH, Hilgers RH, De Mey JG, et al. (2007) Twophoton microscopy of vital murine elastic and muscular arteries. Combined structural and functional imaging with subcellular resolution. $J$ Vasc Res 44: 87-98.[Crossref]

11. Maksimenko AV, Turashev AD(2014) Endothelial glycocalyx of blood circulation system. II. Biological functions, state under normal and pathological conditions, and bioengineering applications. Russ J BioorgKhim 40: 237-251. [Crossref]

12. Nieuwdorp M, Menwese MC, Mooij HL, Ince C, Broekhuizen LN, et al. (2008) Measuring endothelial glycocalyx dimensions in humans: a potential novel tool to monitor vascular vulnerability. J ApplPhysiol 104: 845-852. [Crossref]

13. denUil CA, Klijn E, Lagrand WK, Brugts JJ, Ince C, et al. (2008) The microcirculation in health and critical disease. ProgCardiovasc Dis 51: 161-170.[Crossref]

14. Nieuwdorp M, Meuwese MC, Vink H, Hoekstra JB, Kastelein JJ, et al. (2005) The endothelial glycocalyx: a potential barrier between health and vascular disease. CurrOpinLipidol16: 507-511.[Crossref]

15. Broekhuizen LN, Mooij HL, Kastelein JJ, Stroes ES, Vink H, et al. (2009) Endothelia glycocalyx as potential diagnostic and therapeutic target in cardiovascular disease. CurrOpinLipidol20: 57-62.[Crossref]

16. Becker BF, Jacob M, Leipert S, Salmon AH, Chappell D (2015) Degradation of the endothelial glycocalyx in clinical settings: searching for the sheddases. $\mathrm{Br} J$ ClinPharmacol80: 389-402.[Crossref]

17. Minari FAC (2014) Medical significance of endothelial glycocalyx. Part 2. Its role in vascular diseases and in diabetic complications. Arch CardiolMex 84: 110-116. [Crossref]

18. Salmon AH, Satchell SC (2012) Endothelial glycocalyx dysfunction in disease: albuminuria and increased microvascular permeability. J Pathol 226: 562-574. [Crossref]

19. Vlahu CA, Lemkes BA, Struijk DG, Koopman MG, Krediet RT, et al. (2012) Damage of the endothelial glycocalyx in dialysis patients. J Am SocNephrol23: 1900-1908. [Crossref]

20. Singh A, Friden V, Dasgupta I, Foster RR, Welsh GI, et al. (2011) High glucose cause dysfunction of the human gromerular endothelial glycocalyx. Am J Physiol Renal Physiol 300: F40-F48. [Crossref]

21. Nieuwdorp M, van Haeften TW, Gouverneur MC, Mooij HL, van Lieshout MA, et al (2006) Loss of endothelial glycocalyx during acute hyperglycemia coincides with endothelial dysfunction and coagulation activation in vivo. Diabetes 55: 480-486. [Crossref]

22. Salmito FT, de Oliveira Neves FM, Meneses GC, de Almeida Leitao R, Martins AM, et al.(2015)Glycocalyx injury in adults with nephritic syndrome: association with endothelial function. ClinChimActa 447: 55-58. [Crossref]

23. Shakya S, Wang Y, Mack JA, Maytin EV (2015) Hyperglycemia-induced changes in hyaluronancontribute to impaired skin wound healing in diabetes: review and perspective. Int J Cell Biol 2015: 1-11.

24. Vlahu CA, Krediet RT (2015) Can plasma hyaluronan and hyaluronidase be used as markers of the endothelial glycocalyx state in patients with kidney disease? AdvPerit Dial 31: 3-6. [Crossref]

25. Miranda S, Armengol G, Le Besnerais M, Levesque H, Benhamou Y (2005)New insight into systemic sclerosis related microcirculatory dysfunction by assessment of sublingual microcirculation and vascular glycocalyx layer. Results from a preliminary study. Microvasc Res 99: 72-77. [Crossref]

26. Miranda $\mathrm{CH}$, de CarvalhoBorgers $\mathrm{M}$, Schmidt A, Marin-Neto JA, Pazin-Filho A (2016) Evaluation of the endothelial glycocalyx damage in patients with acute coronary syndrome. Atherosclerosis 247: 184-188. [Crossref]

27. McGee M, Wagner WD (2003) Chondroitin sulfate anticoagulant activity is linked to water transfer. Relevance to proteoglycans structure in atherosclerosis. ArteriosclerThrombVascBiol 23: 1921-1927. [Crossref]

28. van den Berg BM, Vink H, Spaan JA (2003) The endothelial glycocalyx protects against myocardial edema. Circ Res 92: 592-594.[Crossref]

29. Dongaonkar RM, Stewart RH, Geissler HJ, Laine GA (2010) Myocardial microvascular permeability, interstitial oedema, and compromised cardiac function. Cardiovasc Res 87: 331-339. [Crossref] 
30. Becker BF, Chappell D, Jacob M (2010) Endothelial glycocalyx and coronary vascular permeability: the fringe benefit. Basic Res Cardiol105: 687-701.[Crossref]

31. Di Cera E (2003) Atherosclerosis: testing the water. ArteriosclerThrombVascBiol23: 1713-1714.[Crossref]

32. Solursh M, Hardingham TE, Hascall VC, Kimura JH (1980) Separate effects of exogenous hyaluronic acid on proteoglycans synthesis and deposition in pericellular matrix by cultured chick embryo limb chondrocytes. DevBiol 75: 121-129. [Crossref]

33. Seneff S, Davidson RM, Lauritzen A, Samsel A, Wainwright G (2015) A novel hypothesis for atherosclerosis as a cholesterol sulfate deficiency syndrome. TheorBiol Med Model 12: 9.[Crossref]

34. Oberleithner H, Wälte M, Kusche-Vihrog K (2015) Sodium renders endothelial cells sticky for red blood cells. Front Physiol6: 188.[Crossref]

35. Oberleithner H, Wilhelmi M (2015) Vascularglycocalyx sodium store - determinant of salt sensitivity? Blood Purif39: 7-10.[Crossref]

36. Lee DH, Dane MJ, van den Berg BM, Boels MG, van Teeffelen JW, et al. (2014) NEO study group. Deeper penetration of erythrocytes into the endothelial glycocalyx is associated with impaired microvascular perfusion. PLoS One 9: e96477. [Crossref]

37. Oberleithner H (2015) Sodium selective erythrocyte glycocalyx and salt sensitivity in man. Pflugers Arch 467: 1319-1325.[Crossref]

38. Dröge W (2002) Free radicals in the physiological control of cell function. Physiol Rev 82: 47-95.[Crossref]

39. Münzel T, Gori T, Bruno RM, Taddei S (2010) Is oxidative stress a therapeutic target in cardiovascular disease? Eur Heart J 31: 2741-2748.[Crossref]

40. Ekelof S, Jensen SE, Rosenberg J, Gögenur I (2014) Reduced oxidative stress in STEMI patients treated by primary percutaneous coronary intervention and with antioxidant therapy: a systematic review. Cardiovasc Drugs Ther 28: 173-181. [Crossref]

41. Vink H, Constantinescu AA, Spaan JA (2000) Oxidized lipoproteins degrade the endothelial surface layer: implication for platelet-endothelial cell adhesion. Circulation 101: 1500-1502. [Crossref]

42. Kumagai R, Lu X, Kassab GS (2009) Role of glycocalyx in flow-induced production of nitric oxide and reactive oxygen species. Free RadicBiol Med 47: 600-607.[Crossref]

43. Holley A, Miller J, Harding S, Larsen P (2014) Prognostic significance of antioxidant enzymes in acute coronary syndromes. Cardiovasc Res 10 (Suppl 1): S142.

44. Tokarz P, Kaamiranta K, Blasiak J (2013) Role of antioxidant enzymes and smal molecular weight antioxidants in the pathogenesis of age-related macular degeneration (AMD). Biogerontology 14: 461-482. [Crossref]

45. Maksimenko AV (2005) Experimental antioxidant biotherapy for protection of the vascular wall by modified forms of superoxide dismutase and catalase. Curr Pharm Design 11: 2007-2016. [Crossref]

46. Maksimenko AV, Vavaev AV (2012) Antioxidant enzymes as potential targets in cardioprotection and treatment of cardiovascular diseases. Enzyme antioxidants: the next stage of pharmacological counterwork to the oxidative stress. Heart Int 7: 14-19. [Crossref]

47. Martinez Mdel C, Afonso SG, Buzaleh AM, Batlle A (2014) Protective action of antioxidants on hepatic damage induced by griseofulvin. ScientificWorldJournal2014: 982358.[Crossref]

48. Hide D, Ortega-Ribera M, Fernandez-Iglesias A, Fondevila C, Salvado MJ, et al. (2014) A novel form of the human manganese superoxide dismutase protects rat and human livers undergoing ischemia and reperfusion injury. ClinSci (London) 127: 527 537. [Crossref]

49. Mansuroğlu B, Derman S, Yaba A, Kızılbey K (2015) Protective effect of chemically modified SOD on lipid peroxidation and antioxidant status in diabetic rats. Int $J$ BiolMacromol72: 79-87.[Crosser]

50. Wang J, Zhang H, Zhang T, Zhang R, Liu R, et al. (2015) Molecular mechanism on cadmium-induced activity changes of catalase and superoxide dismutase. Int $J$ BiolMacromol77: 59-67.[Crossref]

51. Hood ED, Chorny M, Greineder CF, Alferiev I, Levy RJ, et al. (2014) Endothelial targeting of nanocarriers loaded with antioxidant enzymes for protection agains vascular oxidative stress and inflammation. Biomaterials 35: 3708-3715. [Crossref]

52. Weissig V, Guzman-Villanueva D (2015) Nanocarrier-based antioxidant therapy: promise or delusion? Expert Opin Drug Deliv12: 1783-1790.[Crossref]

53. Richard PU, Duskey JT, Stolarov S, Spulber M, Palivan CG (2015)New concepts to fight oxidative stress: nanosized three-dimensional supramolecular antioxidant assembles. Expert Opin Drug Deliv 12: 1527-1545. [Crossref]
54. Lewis DR, Petersen LK, York AW, Ahuja S, Chae H, et al. (2016) Nanotherapeutics for inhibition of atherogenesis and modulation of inflammation in atherosclerotic plaques. Cardiovasc Res 109: 283-293.[Crossref]

55. Yan F, Mu Y, Yan G, Liu J, Shen J, et al. (2010) Antioxidant enzyme mimics with synergism. Mini Rev Med Chem 10: 342-356.[Crossref]

56. Maksimenko AV (2016) Widening and elaboration of consecutive research into therapeutic antioxidant enzyme derivatives. Oxid Med Cell Longevity2016: 3075695. [Crossref]

57. Maksimenko AV, Golubykh VL, Tischenko EG (2004)The combination of modified antioxidant enzymes for anti-thrombotic protection of the vascular wall: the significance of covalent connection of superoxide dismutase and catalase activities. $J$ Pharm Pharmacol 56: 1463-1468. [Crossref]

58. Ylä-Herttuala S, Sumuvuori H, Karkola K, Möttönen M, Nikkari T (1986) Glycosaminoglycans in normal and atherosclerotic human coronary arteries. Lab Invest 54: 402-407.[Crossref]

59. Kolodgie FD, Burke AP, Farb A, Weber DK, Kutys R, et al. (2002) Differential accumulation of proteoglycans and hyaluronan in culprit lesion: insight into plaque erosion. ArteriosclerThrombVascBiol22: 1642-1648. [Crossref]

60. Wight TN, Merrilees MJ (2004) Proteoglycans in atherosclerosis and restenosis: key roles for versican. Circ Res 94: 1158-1167.[Crossref]

61. Luangwattananun P, Yainoy S, Eiamphungporn W, Songtawee N, Bülow L, et al (2016) Engineering of a novel tri-functional enzyme with MnSOD, catalase and cellpermeable activities. Int J BiolMacromol85: 451-459.[Crossref]

62. Karaduleva EV, Mubarakshina EK, Sharapov MG, Volkova AE, Pimenov OY, et al. (2016)Cardioprotective effect of modified peroxiredoxins in retrograde perfusion of isolated rat heart under conditions of oxidative stress. Bull ExpBiol Med 160: 639-642. [Crossref]

63. Pantrelli G, Halliday I, Spencer TJ, Konig CS, Cöllins MW (2015)Modelling the glycocalyx-endothelium-erythrocyte interaction in the microcirculation: a computational study. ComputMetodsBiomech Biomed Engin 18: 351-361. [Crossref]

64. Grant OC, Tessier MB, Meche L, Mahal LK, Foley BL, et al. (2016) Combining 3D structure with glycan array data provides insight into the origin of glycan specificity. Glycobiology 26: 772-783. [Crossref]

65. Agostino M, Gandhi NS, Mancera RL (2014) Development and application of site mapping methods for the design of glycosaminoglycans. Glycobiology24: 840-851. [Crossref]

66. Sankaranarayanan NV, Desai UR (2014)Toward a robust computational screening strategy for identifying glycosaminoglycan sequences that display high specificity for target proteins. Glycobiology 2: 1323-1333. [Crossref]

67. Jayakanthan M, Jubendradass R, D'Cruz SC, Mathur PP (2015) A use of homology modeling and molecular docking methods: to explore binding mechanisms of nonylphenol and bisphenol A with antioxidant enzymes. Methods MolBiol1268: 273289. [Crossref]

68. Maksimenko A, Turashev A, Fedorovich A, Rogoza A, Tischenko E (2013) Hyaluronidase proof for endothelial glycocalyx as partaker of microcirculation disturbances. J Life Sci7: 171-188.

69. Maksimenko AV, Turashev AD (2012) No-reflow phenomenon and endothelial glycocalyx of microcirculation. Biochem Res Int2012: 859231.[Crossref]

70. Maksimenko AV, Turashev AD, Beabealashvili RS (2015) Stratification of chondroitin sulfate binding sites in 3D-model of bovine testicular hyaluronidase and effective size of glycosaminoglycan coat of the modified protein. Biochemistry (Moscow) 80: 284295. [Crossref]

71. Chao KL, Muthukumar L, Herzberg O(2007) Structure of human hyaluronidasea hyaluronan hydrolyzing enzyme involved in tumor growth and angiogenesis. Biochemistry 46: 6911-6920. [Crossref]

72. Tatara Y, Kakizaki I, Suto S, Ishioka A, Negushi M, et al. (2015) Chondroitin sulfate cluster of epiphycan from salmon nasal cartilage defines binding specificity to collagens. Glycobiology 25: 557-569. [Crossref]

Copyright: (C2017 Maksimenko AV. This is an open-access article distributed under the terms of the Creative Commons Attribution License, which permits unrestricted use, distribution, and reproduction in any medium, provided the original author and source are credited. 